\title{
On the Equilibrium Without Loss in the Discrete Time Models of Economic Dynamics
}

\section{Sabir Isa Hamidov}

Department of Mathematics Cybernetics, Baku State University, Baku, Azerbaijan

\section{Email address:}

sabir818@yahoo.com

\section{To cite this article:}

Sabir Isa Hamidov. On the Equilibrium Without Loss in the Discrete Time Models of Economic Dynamics. International Journal of Theoretical and Applied Mathematics. Vol. 3, No. 6, 2017, pp. 203-209. doi: 10.11648/j.jitam.20170306.15

Received: August 8, 2017; Accepted: November 9, 2017; Published: December 13, 2017

\begin{abstract}
The model of economic dynamics with a fixed budget is considered. The conditions are derived under which the model with a fixed budget has an equilibrium state with the equilibrium prices. The necessary and sufficient conditions for the existence of equilibrium prices are found.
\end{abstract}

Keywords: Production Function, Equilibrium State, Growth Rate

\section{Introduction}

Let's consider a model defined at the moment $t$ by the productive mapping $a(x)[1-3,10]$ :

$$
\begin{gathered}
a(x)=\left\{\tilde{x}=\left(\tilde{x}^{1}, \ldots, \tilde{x}^{n \cdot}\right) \in\left(R_{+}^{n}\right)^{n} \mid 0 \leq \sum_{i=1}^{n} \tilde{x}^{i \cdot} \leq\right. \\
\leq \sum_{i=1}^{n} B^{k} \cdot x^{k \cdot}+\left(F^{1}\left(x^{1 \cdot}\right), \ldots, F^{n}\left(x^{n \cdot}\right)\right), x^{k} . \\
\left.=\left(x^{k 1}, \ldots, x^{k n}\right), k=\overline{1, n}\right\},
\end{gathered}
$$

where $x=\left(x^{1 \cdot}, \ldots, x^{n \cdot}\right) \in\left(R_{+}^{n}\right)^{n}, B^{k}$ is a diagonal matrix, the main diagonal of which has a form

$$
\begin{aligned}
& \left(v^{k 1}, \ldots, v^{k n}\right), v^{k i} \in[0,1](k, i=\overline{1, n}), \\
& F^{j}(x)=\min _{i=1, n} \frac{x^{i}}{c^{i j}}, c^{i j}>0(i, j=\overline{1, n}) .
\end{aligned}
$$

The productive mapping $a^{k}$ of the branch $k$ is in the form

$$
a^{k}(x)=\left\langle 0, B^{k} x+\left(0, \ldots, 0, F^{k}(x), 0, \ldots, 0\right)\right\rangle\left(x \in R_{+}^{n}\right) .
$$

Let's define the conditions under which the model with fixed budgets

$$
M=\{y, U(\ell), \Omega\}
$$

has an equilibrium state $\left(P, x^{1}, \ldots, x^{n}, y\right)$ with equilibrium prices $P=\left(P^{1}, \ldots, P^{n}\right)$. Here as usual $U(\ell)=\left(U^{1}(\ell, ;), \ldots, U^{n}(\ell ;)\right), \quad$ in its turn $U^{i}(\ell, x)=$ $\left[\ell, B^{k} x\right]+\ell^{i} \cdot F^{k}(x) ; \Omega=\left(\lambda^{1}, \ldots, \lambda^{n}\right)$ is a budget vector, $\ell=\left(\ell^{1}, \ldots, \ell^{n}\right)$ is a prices vector.

Let $I=\{1,2, \ldots, n\}$.

The following sets are introduced in [4]

$$
\begin{gathered}
I_{1}(x)=\left\{i \in I \mid x^{i}=0\right\} . \\
I_{2}(x)=\left\{i \in I \mid x^{i}>0\right\}, \\
R^{k}(x)=\left\{i \in I \mid \frac{x^{i}}{c^{i k}}=\min \frac{x^{j}}{c^{j k}}\right\},(k \in I), \\
Q^{k}(x)=I \backslash R^{k}(x), k \in I .
\end{gathered}
$$

The problem of $k-t h$ consumer is as follows:

$$
U^{k}(\ell, x) \rightarrow \max , x \in V=\{x \geq 0,[P, x]=1\} .
$$

Let $\bar{x}^{k \cdot}$ be a maximum point in (1) $(k \in I)$.

\section{Main Part}

Definition. The equilibrium $\left(P, \bar{x}^{1 \cdot}, \ldots, \bar{x}^{n}, \Omega, y\right)$ is called equilibrium without loss if for all $k \in I$ is valid

$$
R^{k}\left(\bar{x}^{k^{\cdot}}\right)=I .
$$


Definition. The prices $P=\left(P^{1}, \ldots, P^{n}\right)$ defined in the equilibrium without loss is called an equilibrium prices without loss.

Note that due to the consequence of the lemma 1 [4] we have $I_{1}\left(\bar{x}^{k}\right)=\emptyset$.

First, consider the consumer problem without loss, and then the equilibrium without loss.

Note that the solution of the $k-t h$ problem without loss has the following property

$$
\bar{x}^{k \cdot}=\gamma^{k} \cdot c^{\cdot k}(k \in I),
$$

where $\gamma^{k} \geq 0$ is some constant that is equal to the value of the $k-t h$ production function in the point $\bar{x}^{k}$.

Indeed from the conditions $R^{k}\left(\bar{x}^{k^{\cdot}}\right)=I, I_{1}\left(\bar{x}^{k^{\cdot}}\right)=\emptyset$ we get that

$$
\frac{\bar{x}^{k 1}}{c^{1 k}}=\cdots=\frac{\bar{x}^{k n}}{c^{n k}}=\gamma^{k}, \bar{x}^{k i}>0(k, i \in I) .
$$

Consider the problem of kth consumer without loss. To analyze the problem (1) we apply the necessary and sufficient conditions for the extremum, wherein in the point $\bar{x}$ the maximum is reached if and only if when

$$
\left(U^{k}\right)^{\prime}(\bar{x}, g) \leq 0 \text { for all } g \in G_{\bar{x}}(V)
$$

where

$$
G_{\bar{x}}(V)=\left\{g \in R^{n} \mid[P, g]=0, g^{i} \geq 0 \forall i \in I_{1}(\bar{x})\right\} .
$$

As is known [2], $\left(U^{k}\right)^{\prime}(\bar{x}, g)=q^{k}(g)$, where

$$
q^{k}(g)=\left[\ell_{v}^{k}, g\right]+\ell^{k} \cdot \min _{i \in R^{k}(\bar{x})} \frac{g^{i}}{c^{i k}}(k \in I) .
$$

Then in our case (without loss) we get that the necessary and sufficient optimality conditions for $\bar{x}$ of the branch $k$ take the forms

$$
\begin{gathered}
q^{k}(g)=\left[\ell_{v}^{k}, g\right]+\ell^{k} \cdot \min _{i \in I} \frac{g^{i}}{c^{i k}} \leq 0 \forall g \in \Omega= \\
\left\{g \in R^{n} \mid[P, g]=0\right\} .
\end{gathered}
$$

Lemma 1. The number $\mu^{k}(k \in I)$, defined in the lemma 4 [4] in the case of without loss $\left(R^{k}(\bar{x})=I\right)$ coincides with the maximal growth rate of the total wealth of the $k-t h$ branch and is equal to

$$
\mu^{k}=\frac{\ell^{k}+\left[\ell_{v}^{k}, c^{\cdot k}\right]}{\left[P, c^{\cdot k}\right]}(k \in I),
$$

where $\ell_{v}^{k}=\left(\ell^{1} \cdot v^{k 1}, \ldots, \ell^{n} \cdot v^{k n}\right)$.

Proof. In the case, when $R^{k}(x)=I$, as follows from lemma 4 [4] the number $\mu^{k}$ is in the form of (4).

From the other hand let $\bar{x}$ be a maximum point in the problem (1) of the kth consumer that is indeed similar to the following relations

$$
\max _{x \geq 0} \frac{U^{k}(\ell, x)}{[P, x]}=\frac{U^{k}(\ell, \bar{x})}{[P, \bar{x}]}(k \in I)
$$

where the functions $U^{k}(\ell, x)$ are defined in the introduction.

From the definition of $\mu^{k}$ and (2) we get that

$$
\max _{x \geq 0} \mu^{k}(x)=\mu^{k},
$$

where $\mu^{k}$ is of the form (4).

Lemma is proved.

Theorem 1 . Let strongly positive vector $P=\left(P^{1}, \ldots, P^{n}\right)$ be given, index $k \in I$ and the number $\mu^{k}$ is defined by (4). The vector $\bar{x}$ is a solution of the problem (1), satisfying the relation

$$
R^{k}(\bar{x})=I
$$

Then and only then

$$
\begin{array}{r}
\ell^{j} \cdot v^{k j} \leq \mu^{k} \cdot P^{j} \forall j \in I, \\
P \in \frac{1}{\mu^{k}} \cdot\left(\ell_{v}^{k}+\partial \tilde{q}^{k}\right),
\end{array}
$$

where $\ell_{v}^{k}, \partial \tilde{q}^{k}$ are defined in the lemma 3 [4] for the case $R^{k}(\bar{x})=I$.

The proof follows from the consequence of 1 [4], theorem 1 [4] and lemma 1

Remark. By given $\mu^{k}(k \in I)$, the equality (3) may be considered as a system of $n$ linear equation with respect to variables- coordinates of equilibrium vector of prices $P$ without loss

$$
\left[P, c^{\cdot k}\right]=\frac{1}{\mu^{k}} \cdot\left[\ell^{k}+\left[\ell_{v}^{k}, c^{\cdot k}\right]\right](k \in I),
$$

where $c^{\cdot k}=\left(c^{1 k}, \ldots, c^{n k}\right), \ell_{v}^{k}=\left(\ell^{1} \cdot v^{k 1}, \ldots, \ell^{n} \cdot v^{k n}\right)$ and in contrary by the given prices $P$ from the equality (3) are defined uniquely the maximal growth rate $\mu^{k}$ of the total wealth of the $k-t h$ branch $(k \in I)$.

Let's consider the following problem. Let $v^{k i} \geq 0, c^{i k}>$ $0(i, k \in I)$ be given. By which $\ell^{i}>0, \mu^{k}>0(i, k \in I)$ there exists the vector $P=\left(P^{1}, \ldots, P^{n}\right)$, that for some $\lambda^{1}, \ldots, \lambda^{n}, y$ is an equilibrium prices without loss in the model $M$, defined by the set $\left\{\ell, \lambda^{1}, \ldots, \lambda^{n}, y\right\}$.

Note that it follows from the lemma 3 [4] that in the case of lossless $\left(R^{k}(\bar{x})=I\right)$ subdifferential $\partial \tilde{q}^{k}$ takes the form $[5,6]$

$$
\begin{gathered}
\partial \tilde{q}^{k}=\left\{f=\ell^{k} \cdot\left(f^{1}, \ldots, f^{n}\right) \mid \exists \alpha^{i} \geq 0: \sum_{i \in I} \alpha^{i}=1, f^{i}=\right. \\
\left.\frac{\ell^{k} \cdot \alpha^{i}}{c^{i k}} \forall i \in I\right\} .
\end{gathered}
$$

It can be shown that for each set $\mu^{1}>0, \ldots, \mu^{n}>0$ there are weights $\lambda^{1}, \ldots, \lambda^{n}$ such that the growth rate in the model defined by the weights $\lambda^{1}, \ldots, \lambda^{n}$, coincides up to those cells in which $\lambda^{i}=0$ and the growth rate does not depend on the choice of the equilibrium price $P$.

Lemma 2. Let the numbers $\ell^{i}>0, \mu^{k}>0, v^{j i} \geq 0, c^{i j}>$ $0(i, j, k \in I)$ be given and $\left(P, x^{1 \cdot}, \ldots, x^{n \cdot}\right)$ is an equilibrium without loss in the model with utility functions $U^{j}$, defined in the introduction section by the budgets $\lambda^{i}=\left[P, x^{j}\right]$ and distributed by the vector $y=\sum_{i=1}^{n} x^{i \cdot}(j \in I)$. The relation (6) is fulfilled for $\forall k \in I$ then and only then when for any 
$v^{j i} \geq 0$ and $u^{j}(i, j \in I)$, satisfying the relations

$$
\sum_{j \in I} \mu^{j} \cdot\left(v^{j i}+u^{j} \cdot c^{i j}\right)=0 \forall i \in I,
$$

the inequality below is valid

$$
\sum_{j \in I}\left(\sum_{i \in I} v^{j i} \cdot \ell^{i} \cdot v^{j i}+u^{j} \cdot\left(\ell^{j}+\sum_{i \in I} \ell^{i} \cdot v^{j i} \cdot c^{i j}\right)\right) \leq 0 .
$$

Proof. It follows from (7) that

$$
\partial \tilde{q}^{k}=\left\{\left(\beta^{1}, \ldots, \beta^{n}\right) \mid \sum_{i \in I} c^{i k} \cdot \beta^{i}=\ell^{k}, \beta^{i} \geq 0\right\}(k \in I) .
$$

Denote the set of the form $\frac{1}{\mu^{k}}\left(\ell_{v}^{k}+\partial \tilde{q}^{k}\right)$ by $\Phi^{k}$, that considering lemma 3 [4] (in the case $R^{k}(\bar{x})=I$ ) indeed has a form

$$
\begin{gathered}
\Phi^{k}=\left\{\frac{1}{\mu^{k}} \cdot\left(\gamma^{1}, \ldots, \gamma^{n}\right) \mid \gamma^{i}=\ell^{i} \cdot v^{k i}+\beta^{i}, \sum_{i \in I} c^{i k} \cdot \beta^{i}\right. \\
\left.=\ell^{k}, \beta^{i} \geq 0\right\}= \\
=\left\{\frac{1}{\mu^{k}} \cdot\left(\gamma^{1}, \ldots, \gamma^{n}\right) \mid \sum_{i \in I} c^{i k} \cdot\left(\gamma^{i}-\ell^{i} \cdot v^{k i}\right)=\ell^{k}, \gamma^{i}\right. \\
\left.\geq \ell^{i} \cdot v^{k i}\right\}= \\
=\left\{\frac{1}{\mu^{k}} \cdot \gamma \mid \sum_{i \in I} c^{i k} \cdot \gamma^{i}=\ell^{k}+\sum_{i \in I} \ell^{i} \cdot v^{k i} \cdot c^{i k}, \gamma^{i}\right. \\
\left.\geq \ell^{i} \cdot v^{k i}\right\}(k \in I) .
\end{gathered}
$$

Since for all $k \in I$ due the conditions of the lemma3 [4] (6) is fulfilled we have

$$
P \in \bigcap_{k \in I} \Phi^{k}, \text { where } P=\left(P^{1}, \ldots, P^{n}\right) .
$$

Thus

$$
\begin{gathered}
\mu^{j} \cdot P^{i} \geq \ell^{i} \cdot v^{j i}(i, j \in I), \\
\mu^{j} \cdot \sum_{i \in I} P^{i} \cdot c^{i j}=\ell^{j}+\sum_{i \in I} \ell^{i} \cdot v^{j i} \cdot c^{i j}(j \in I) .
\end{gathered}
$$

This system may be written as a system of inequalities

$$
\left\{\begin{array}{c}
\mu^{j} \cdot P^{i} \geq \ell^{i} \cdot v^{j i}(i, j \in I), \\
\mu^{j} \cdot \sum_{i \in I} P^{i} \cdot c^{i j} \geq \ell^{j}+\sum_{i \in I} \ell^{i} \cdot v^{j i} \cdot c^{i j} \quad(j \in I), \\
-\mu^{j} \cdot \sum_{i \in I} P^{i} \cdot c^{i j} \geq-\left(\ell^{j}+\sum_{i \in I} \ell^{i} \cdot v^{j i} \cdot c^{i j}\right)(j \in I) .
\end{array}\right.
$$

Let's introduce the denotations

$$
\left\{\begin{array}{c}
f^{j i}=e^{i} \cdot \mu^{j}, \\
f^{j}=\mu^{j} \cdot\left(c^{1 j}, c^{2 j}, \ldots, c^{n j}\right), \\
f^{n+j}=-f^{j}, \\
\beta^{j i}=\ell^{i} \cdot v^{j i},(i, j \in I), \\
\beta^{j}=\ell^{j}+\sum_{i \in I} \ell^{i} \cdot v^{j i} \cdot c^{i j}, \\
\beta^{n+j}=-\beta^{j},
\end{array}\right.
$$

where $e^{i}$ is $i-t h$ ort in the space $R_{+}^{n}$.

Rewriting the system (10) in new denotation considering (11) we obtain

$$
\left\{\begin{array}{c}
{\left[f^{j i}, P\right] \geq \beta^{j i}(i, j \in I)} \\
{\left[f^{j}, P\right] \geq \beta^{j}(j \in I)} \\
{\left[f^{n+j}, P\right] \geq \beta^{n+j}(j \in I) .}
\end{array}\right.
$$

Then, by the theorem of [7] for the compatibility of the system (12) is necessary and sufficient that for any $v^{j i} \geq$ $0, v^{j} \geq 0, v^{n+j} \geq 0(i, j \in I)$ from the equality

$$
\sum_{i, j \in I} v^{j i} \cdot f^{j i}+\sum_{j \in I} v^{j} \cdot f^{j}+\sum_{j \in I} v^{n+j} \cdot f^{n+j}=0
$$

follow the inequality

$$
\sum_{i, j \in I} v^{j i} \cdot \beta^{j i}+\sum_{j \in I} v^{j} \cdot \beta^{j}+\sum_{j \in I} v^{n+j} \cdot \beta^{n+j} \leq 0 .
$$

In our case necessary and sufficient conditions have a form

$$
\begin{gathered}
\sum_{j \in I} \mu^{j} \cdot\left(v^{j i}+\left(v^{j}-v^{n+j}\right) \cdot c^{i j}\right)=0 \text { for } \forall i \in I, \\
\sum_{j \in I}\left(\sum_{i \in I} v^{j i} \cdot \ell^{i} \cdot v^{j i}+\left(v^{j}-v^{n+j}\right) \cdot\left(\ell^{j}+\sum_{i \in I} \ell^{i} \cdot v^{j i} .\right.\right. \\
\left.\left.c^{i j}\right)\right) \leq 0 .
\end{gathered}
$$

Let $u^{j}=v^{j}-v^{n+j}, \forall i \in I$.

Then it follows from (13) that $u^{j}$ are such that

$$
\sum_{j \in I} \mu^{j} \cdot u^{j} \cdot c^{i j} \leq 0 \text { for } \forall i \in I .
$$

Substituting $u^{j}$ ( $j \in I$ ) into (13) we get (8), (9).

Lemma is proved.

Consider the matrix $C=\left(c^{i j}\right)_{i, j=1}^{n}$. Let $|C|$ be its determinant. It is valid Lemma 3 . Let the numbers $v^{j i} \geq$ $0, c^{i j}>0(i, j \in I)$ be given and $|C| \neq 0$. The following conditions are equivalent

1. numbers $v^{j i} \geq 0, u^{j}(i, j \in I)$ are such that (8) and (9) are fulfilled;

2. numbers $\ell^{i}, \mu^{j}(i, j \in I)$ are such that for $\forall i, j \in I$ is valid

$$
\begin{gathered}
\ell^{i} \cdot v^{j i}+\frac{1}{|C|} \cdot \sum_{i \in I}(-1)^{i+k+1} \cdot \frac{\mu^{j}}{\mu^{k}}\left(\ell^{k}+\sum_{m \in I} \ell^{m} \cdot v^{k m} .\right. \\
\left.c^{m k}\right)\left|C_{i}^{k}\right| \leq 0,
\end{gathered}
$$

where $C_{i}^{k}$ is $(n-1) \times(n-1)$ matrix obtained from the matrix $C$ by removing $k-t h$ column and $i-t h$ row. 
Proof. The system of equalities (8) we rewrite as follows

$$
\sum_{j \in I} \mu^{j} \cdot u^{j} \cdot c^{i j}=-\sum_{j \in I} \mu^{j} \cdot v^{j i}, \forall i \in I .
$$

Consider the equation

$$
A \cdot u=-b
$$

where

$$
\begin{gathered}
A=\left(\begin{array}{cccc}
\mu^{1} \cdot c^{11} & \mu^{2} \cdot c^{12} & \ldots & \mu^{n} \cdot c^{1 n} \\
\mu^{1} \cdot c^{21} & \mu^{2} \cdot c^{22} & \ldots & \mu^{n} \cdot c^{2 n} \\
\cdots & \cdots & \ldots & \cdots \\
\mu^{1} \cdot c^{n 1} & \mu^{2} \cdot c^{n 2} & \ldots & \mu^{n} \cdot c^{n n}
\end{array}\right), b=\left(\begin{array}{c}
b^{1} \\
b^{2} \\
\ldots \\
b^{n}
\end{array}\right), \\
b^{m}=\sum_{k \in I} \mu^{k} \cdot v^{k m}(m \in I) .
\end{gathered}
$$

Note that

$$
|A|=\prod_{j \in I} \mu^{j} \cdot|C|,
$$

where $|C|$ is a determinant of the matrix.

As is known [8] the equation (16) has a solution (note that $|C| \neq 0)$ :

$$
u^{j}=-\frac{\left|A^{j}\right|}{|A|}(j \in I)
$$

where $A^{j}$ is $n \times n$ matrix obtained from the matrix $A$ by replacing $j-t h$ column by the row $b$.

Expanding the determinant of the matrix $A^{j}$ over the element of the $j-t h$ column ( $\mathrm{t}$ refers the column $b$ ), we get

$$
\begin{aligned}
\left|A^{j}\right|=\frac{1}{\mu^{j}} \cdot \prod_{j \in I} \mu^{k} & \cdot\left|C^{j}\right| \\
& =\frac{1}{\mu^{j}} \\
& \cdot \prod_{j \in I} \mu^{k} \cdot \sum_{m \in I}(-1)^{m+j} \cdot b^{m} \cdot\left|C_{m}^{j}\right|(j \in I),
\end{aligned}
$$

here $C_{m}^{j}$ is $(n-1) \times(n-1)$ matrix obtained from the matrix $C$ by removing $j-t h$ column and $m-t h$ row.

Substituting the values of $|A|,\left|A^{j}\right|$ into the solution of the equation (16), we obtain

$$
u^{j}=\frac{1}{\mu^{j} \cdot|C|} \cdot \sum_{m \in I}(-1)^{m+j+1} \cdot\left(\sum_{k \in I} \mu^{k} \cdot v^{k m}\right) \cdot\left|C_{m}^{j}\right|(j \in I) .
$$

Note that (16) is indeed matrix form of the system of equations (15) relatively $u$.

Then

$$
\sum_{j \in I}\left(\sum_{i \in I} v^{j i} \cdot \ell^{i} \cdot v^{j i}+u^{j} \cdot\left(\ell^{j}+\sum_{i \in I} \ell^{i} \cdot v^{j i} \cdot c^{i j}\right)\right)=
$$

$$
\begin{gathered}
=\sum_{j \in I}\left[\sum_{i \in I} v^{j i} \cdot \ell^{i} \cdot v^{j i}+\frac{1}{\mu^{j} \cdot|C|} \cdot\left(\ell^{j}+\sum_{i \in I} \ell^{i} \cdot v^{j i} \cdot c^{i j}\right) \times\right. \\
\left.\times \sum_{m \in I}(-1)^{m+j+1} \cdot\left(\sum_{k \in I} \mu^{k} \cdot v^{k m}\right) \cdot\left|C_{m}^{j}\right|\right]
\end{gathered}
$$

where $|C| \neq 0$.

The expression (17) after some transformations may be reduced to the form

$$
\sum_{j \in I} \sum_{i \in I} d^{j i} \cdot v^{j i}=[d, v],
$$

where $d=\left(d^{11}, d^{12}, \ldots, d^{n n}\right), v=\left(v^{11}, v^{12}, \ldots, v^{n n}\right)$, and $d^{j i}$ are corresponding coefficients at $v^{j i}$, depending on $\ell^{i}, v^{j i}, c^{i j}, \mu^{j}$ :

$$
d^{j i}=d^{j i}\left(\ell^{i}, v^{j i}, c^{i j}, \mu^{j}\right)(i, j \in I) .
$$

To reduce the expression (19) to the form (19), it is necessary in (18) pass from $v^{k m}$ to $v^{j i}$. To do this we accept reindexing " $k " \leftrightarrow " j ", " m " \leftrightarrow " i "$ :

$$
\begin{aligned}
& \sum_{j \in I} \frac{1}{\mu^{j} \cdot|C|} \cdot\left(\ell^{j}+\sum_{i \in I} \ell^{i} \cdot v^{j i} \cdot c^{i j}\right) \\
& \cdot \sum_{m \in I}(-1)^{m+j+1} \cdot\left(\sum_{k \in I} \mu^{k} \cdot v^{k m}\right) \cdot\left|C_{m}^{j}\right|= \\
& =\sum_{k \in I} \frac{1}{\mu^{j} \cdot|C|} \cdot\left(\ell^{k}+\sum_{m \in I} \ell^{m} \cdot v^{k m} \cdot c^{m k}\right) \\
& \cdot \sum_{m \in I}(-1)^{i+k+1} \cdot\left(\sum_{j \in I} \mu^{j} \cdot v^{j i}\right) \times \\
& \times\left|C_{i}^{k}\right|=\sum_{j \in I} \frac{1}{|C|} \cdot \sum_{k \in I} \frac{\mu^{j}}{\mu^{k}}\left(\ell^{k}{ }^{k}\right. \\
& \left.+\sum_{m \in I} \ell^{m} \cdot v^{k m} \cdot c^{m k}\right) \sum_{i \in I}(-1)^{i+k+1} \\
& \cdot \mid C_{i}^{k \mid} \cdot v^{j i}= \\
& =\sum_{j \in I} \sum_{i \in I} \frac{1}{|C|} \cdot \sum_{k \in I}(-1)^{i+k+1} \cdot \frac{\mu^{j}}{\mu^{k}} \\
& \left(\ell^{k}+\sum_{m \in I} \ell^{m} \cdot v^{k m} \cdot c^{m k}\right) \cdot\left|C_{i}^{k}\right| \cdot v^{j i}
\end{aligned}
$$

Then (18) turns to

$$
\begin{gathered}
\sum_{j \in I} \sum_{i \in I}\left(\ell^{i} \cdot v^{j i}+\frac{1}{|C|} \cdot \sum_{k \in I}(-1)^{i+k+1} \times\right. \\
\left.\times \frac{\mu^{j}}{\mu^{k}} \cdot\left(\ell^{k}+\sum_{m \in I} \ell^{m} \cdot v^{k m} \cdot c^{m k}\right) \cdot\left|C_{i}^{k}\right| \cdot v^{j i}\right) .
\end{gathered}
$$

Thus comparing the last one with (19), we arrive to 


$$
\begin{gathered}
d^{j i}=\ell^{i} \cdot v^{j i}+\frac{1}{|C|} \cdot \sum_{k \in I}(-1)^{i+k+1} \cdot \frac{\mu^{j}}{\mu^{k}} \cdot\left(\ell^{k}+\sum_{m \in I} \ell^{m} .\right. \\
\left.v^{k m} \cdot c^{m k}\right) \cdot\left|C_{i}^{k}\right| .
\end{gathered}
$$

Then the condition (9) is equivalent to

$$
[d, v] \leq 0,
$$

where scalar product $[d, v]$ is defined by the formula (19). It does not exist $v=\left(v^{11}, v^{12}, \ldots, v^{1 n}\right)$ such that $[d, v]>0$ then and only then when тогда и all $d^{j i} \leq 0$ for $\forall i, j \in I$, where $d^{j i}$ is defined by (20).

Lemma is proved.

It takes place

Theorem 2. Let the numbers $v^{j i} \geq 0$ и $c^{i j}>0(i, j \in I)$ are such that $\max _{j \in I} v^{j i}>0$ и $|C| \neq 0$. The equilibrium prices without loss by given $v^{j i}, c^{j i}$ and some $\ell^{i}>0(i \in I)$ and $\mu^{j}>0(j \in I)$ exist only and only when (14) is satisfied; the coefficients $\mu^{k}(k \in I)$ and equilibrium prices $P$ are related by the formula

$$
\mu^{k} \cdot\left[P, c^{\cdot k}\right]=\ell^{k}+\left[\ell_{v}^{k}, c^{\cdot k}\right](k \in I),
$$

where $c^{\cdot k}=\left(c^{1 k}, \ldots, c^{n k}\right), \ell_{v}^{k}=\left(\ell^{1} \cdot v^{k 1}, \ldots, \ell^{n} \cdot v^{k n}\right)$.

The proof immediately follows from the lemmas 2 and 3.

Note 1 . Note that the condition (14), that is necessary and sufficient condition of existence equilibrium prices without loss does not depend on the vector of distributed resources $y$.

Note 2. By given $v^{j i} \geq 0, c^{i j}>0(i, j \in I)$ the parameters $\ell^{i}, \mu^{j}(i, j \in I)$ is a solution of the system of inequalities (14).

Example. Rewrite the conditions (14) for the cases $n=2, n=3$ и $v^{j i}=0, \mu^{i}=\mu^{j}(i \neq j)(i, j \in I)$.

a) by $n=2$ we have

$$
\begin{gathered}
\frac{1}{|C|} \cdot\left[-\left(c^{22}+v^{11} \cdot c^{21} \cdot c^{12}\right) \cdot \ell^{1}+\left(1+v^{22} \cdot c^{22}\right) \cdot c^{21}\right. \\
\left.\cdot \ell^{2}\right] \leq 0
\end{gathered}
$$$$
\frac{1}{|C|} \cdot\left[-\left(1+v^{11} \cdot c^{11}\right) \cdot c^{22} \cdot \ell^{1}+\left(1+v^{22} \cdot c^{22}\right) \cdot c^{21} \cdot \ell^{2}\right]
$$$$
\leq 0
$$

$$
\begin{gathered}
\frac{1}{|C|} \cdot\left[\left(1+v^{11} \cdot c^{11}\right) \cdot c^{12} \cdot \ell^{1}-\left(1+v^{22} \cdot c^{22}\right) \cdot c^{11} \cdot \ell^{2}\right] \\
\leq 0,
\end{gathered}
$$$$
\frac{1}{|C|} \cdot\left[\left(1+v^{11} \cdot c^{11}\right) \cdot c^{12} \cdot \ell^{1}-\left(c^{11}+v^{22} \cdot c^{12} \cdot c^{21}\right) \cdot \ell^{2}\right]
$$

$$
\leq 0 \text {, }
$$

where $|C|$ is a determinant of the $2 \times 2$ matrix $C$;

b) by $n=3$ conditions (14) turn to:

$$
\begin{gathered}
\left(v^{11}-\frac{\left|C_{1}^{1}\right|}{|C|} \cdot\left(1+v^{11} \cdot c^{11}\right)\right) \cdot \ell^{1}+\frac{\left|C_{1}^{1}\right|}{|C|} \cdot\left(1+v^{22} \cdot c^{22}\right) \\
\cdot \ell^{2}-
\end{gathered}
$$

$$
\begin{gathered}
-\frac{\left|C_{1}^{3}\right|}{|C|} \cdot\left(1+v^{33} \cdot c^{33}\right) \cdot \ell^{3} \leq 0 \\
\frac{1}{|C|} \cdot\left[-\left(1+v^{11} \cdot c^{11}\right) \cdot\left|C_{1}^{1}\right| \cdot \ell^{1}+\left(1+v^{22} \cdot c^{22}\right) \cdot\left|C_{1}^{2}\right| \cdot \ell^{2}\right. \\
- \\
\left.-\left(1+v^{33} \cdot c^{33}\right) \cdot\left|C_{1}^{3}\right| \cdot \ell^{3}\right] \leq 0 \\
\frac{1}{|C|} \cdot\left[\left(1+v^{11} \cdot c^{11}\right) \cdot\left|C_{2}^{1}\right| \cdot \ell^{1}-\left(1+v^{22} \cdot c^{22}\right) \cdot\left|C_{2}^{2}\right| \cdot \ell^{2}-\right. \\
\left.-\left(1+v^{33} \cdot c^{33}\right) \cdot\left|C_{2}^{3}\right| \cdot \ell^{3}\right] \leq 0 \\
\frac{\left|C_{2}^{1}\right|}{|C|} \cdot\left(1+v^{11} \cdot c^{11}\right) \cdot \ell^{1}+\left(v^{22}-\frac{\left|C_{2}^{2}\right|}{|C|} \cdot\left(1+v^{22} \cdot c^{22}\right)\right) \\
\cdot \ell^{2}+ \\
+\frac{\left|C_{2}^{3}\right|}{|C|} \cdot\left(1+v^{33} \cdot c^{33}\right) \cdot \ell^{3} \leq 0 \\
\frac{1}{|C|} \cdot\left[-\left(1+v^{11} \cdot c^{11}\right) \cdot\left|C_{3}^{1}\right| \cdot \ell^{1}+\left(1+v^{22} \cdot c^{22}\right) \cdot\left|C_{3}^{2}\right| \cdot \ell^{2}\right. \\
- \\
\left.-\left(1+v^{33} \cdot c^{33}\right) \cdot\left|C_{3}^{3}\right| \cdot \ell^{3}\right] \leq 0 \\
-\frac{\left|C_{3}^{1}\right|}{|C|} \cdot\left(1+v^{11} \cdot c^{11}\right) \cdot \ell^{1}+\frac{\left|C_{3}^{2}\right|}{|C|} \cdot\left(1+v^{22} \cdot c^{22}\right) \cdot \ell^{2}+ \\
+\left(v^{33}-\frac{\left|C_{3}^{3}\right|}{|C|} \cdot\left(1+v^{33} \cdot c^{33}\right)\right) \cdot \ell^{3} \leq 0, \\
\end{gathered}
$$

where $|C|$ is a determinant of the $3 \times 3$ matrix $C, \mathrm{C}_{j}^{i}$ is $2 \times 2$ matrix obtained from $C$ by removing $i-t h$ column and $j$-th row.

Introduce the numbers

$$
=\left\{\begin{array}{l}
\ell^{i} \cdot v^{j i}+(-1)^{i+j+1} \cdot \frac{\left|C_{i}^{j}\right|}{|C|} \cdot\left(\ell^{j}+\sum_{m \in I} \ell^{m} \cdot v^{j m} \cdot c^{m j}\right), \text { if } k=j, \\
(-1)^{i+k+1} \cdot \frac{\left|C_{i}^{k}\right|}{|C|} \cdot\left(\ell^{k}+\sum_{m \in I} \ell^{m} \cdot v^{k m} \cdot c^{m k}\right), \text { if } k \neq j,(i, j, k \in I)
\end{array}\right.
$$

and vector $\tilde{d}^{k j}$ :

$$
\tilde{d}^{k j}=\left(\begin{array}{c}
-d_{1}^{k j} \\
\vdots \\
\vdots \\
-d_{n}^{k j}
\end{array}\right)(k, j \in I)
$$

Proposition 2. The numbers $\mu^{j}>0(j \in I)$, satisfying (14), exist if and only if when there exists the index $k_{o} \in I$ and $æ^{k}$ such that

$$
\mathfrak{x}^{k_{o}}>0, \sum_{k=1}^{n^{2}} \mathfrak{x}^{k} \cdot \tilde{d}^{k j} \geq 0(j \in I),
$$

where $\tilde{d}^{k j}$ is defined by (22).

Proof. Necessity. Let there exist the numbers $\mu^{j}>$ 
$0(j \in I)$, satisfying the condition (14) that may be written as

$$
\begin{aligned}
\ell^{i} \cdot v^{j i} \cdot \frac{1}{\mu^{j}}+\frac{1}{|C|} & \cdot \sum_{k \in I}(-1)^{i+k+1} \\
& \cdot\left(\ell^{k}+\sum_{m \in I} \ell^{m} \cdot v^{k m} \cdot c^{m k}\right) \cdot\left|C_{i}^{k}\right| \cdot \frac{1}{\mu^{k}} \leq 0
\end{aligned}
$$

$$
(i, j \in I) \text {. }
$$

Let's rewrite (24) in more proper form

$$
\begin{gathered}
\frac{1}{|C|} \cdot \sum_{k \in I \backslash\{j\}}(-1)^{i+k+1} \cdot\left(\ell^{k}+\sum_{m \in I} \ell^{m} \cdot v^{k m} \cdot c^{m k}\right) \cdot\left|C_{i}^{k}\right| \cdot \\
\frac{1}{\mu^{k}}+ \\
+\left[\ell^{i} \cdot v^{j i}+\frac{1}{|C|} \cdot(-1)^{i+j+1} \cdot\left(\ell^{j}+\sum_{m \in I} \ell^{m} \cdot v^{j m} \cdot c^{m j}\right)\right. \\
\left.\cdot\left|C_{i}^{j}\right|\right] \cdot \frac{1}{\mu^{j}} \leq \leq 0(i, j \in I) .
\end{gathered}
$$

Let $\tilde{\mu}=\left(\frac{1}{\mu^{1}}, \ldots, \frac{1}{\mu^{n}}\right), B$ be a matrix the elements of which are corresponding coefficients at $\frac{1}{\mu^{j}}(j \in I)$ in (25).

Then (25) takes the form

- there exists $\tilde{\mu} \gg 0$ such that $B \cdot \tilde{\mu} \leq 0$. Let $D=-B$. Then there exists the vector $\tilde{\mu} \gg 0$ such that $D \cdot \tilde{\mu} \geq 0$, where

$$
D=\left(\begin{array}{c}
-D_{1} \\
-D_{2} \\
\vdots \\
-D_{n}
\end{array}\right) D_{i}=\left(d_{i}^{k j}\right)_{k, j=1}^{n}(i \in I)
$$

For $n^{2} \times n$ matrix $D$ introduce the cone

$$
K=\{\tilde{\mu} \mid D \cdot \tilde{\mu} \geq 0\} .
$$

As follows from (26)

$$
K \cap \operatorname{int} R_{+}^{n} \neq \varnothing .
$$

Assume the contrary, i. e.

$$
K \cap \operatorname{int} R_{+}^{n}=\varnothing .
$$

Therefore, these two sets can be identified: a vector $f=$ $\left(f^{1}, \ldots, f^{n}\right)$ such that

$$
\left\{\begin{array}{c}
{[f, \mu] \leq 0, \text { if } \mu \in K,} \\
{[f, \mu]>0, \text { if } \mu \in R_{+}^{n} .}
\end{array}\right.
$$

From this and the second inequality follows that $f>0$. Thus there exists the vector $f>0$ such that $[f, \tilde{\mu}] \leq 0$, if $D \cdot \tilde{\mu} \geq 0$ or $\left[d^{i}, \tilde{\mu}\right] \geq 0 i=\overline{1, n^{2}}$, where $d^{i}$ is a row of the matrix $D$. Then the vector $f$ takes the form

$$
f=\sum_{i=1}^{n^{2}} æ^{i} \cdot d^{i}, æ^{i} \leq 0 .
$$

Rewrite the cone $K$ as follows

$$
K=\{\tilde{\mu} \mid D \cdot \tilde{\mu} \geq 0 \forall i \in I\} .
$$

In this case from (26) follows that

$$
f \in-K^{*},
$$

where $K^{*}$ is an adjoint cone.

The proposition (28) assumes there exists $æ^{i} \leq 0$ for which

$$
\sum_{i=1}^{n^{2}} æ^{i} \cdot d^{i} \leq 0 .
$$

In its turn (26) means that there does not exist $æ^{i} \leq 0$ such that $\sum_{i=1}^{n^{2}} æ^{i} \cdot d^{i} \geq 0$, i. e. if $\sum_{i=1}^{n^{2}} æ^{i} \cdot d^{i} \geq 0$, then there exists the index $i_{o} \in I$ such that $æ^{i_{o}}>0$.

Sufficiency. Let (23) be valid. Introduce the cone $K$ by the formula (27) adjoint cone $K^{*}$. Making the same reasoning as above, but in reverse order, it is easy to show that there exist $\mu^{j}>0(j \in I)$ satisfying (14).

Propostion is proved.

Note. The system of $n^{2}$ linear inequalities (24) with respect to the variables $\ell^{i}, v^{j i}, c^{i j}, \mu^{j}$ may be reduced to the system of $n$ superlinear inequalities with respect to the same variables.

For this purpose let's introduce the denotations

$$
\begin{gathered}
d_{i}^{j}=(-1)^{i+j+1} \cdot \frac{\left|C_{i}^{j}\right|}{|C|} \cdot\left(\ell^{j}+\sum_{m \in I} \ell^{m} \cdot v^{j m} \cdot c^{m j}\right)(i, j \in I), \\
d_{i}=\left(d_{i}^{1}, \ldots, d_{i}^{n}\right)(i \in I) .
\end{gathered}
$$

Then the inequalities (24) may be rewritten as

$$
\left\{\begin{array}{c}
{\left[d_{1}, \tilde{\mu}\right] \leq-\ell^{1} \cdot v^{1 i} \cdot \frac{1}{\mu^{i}} \forall i \in I,} \\
\cdots \cdots \cdots \cdots \cdots \cdots \cdots \cdots \cdots \cdots \cdots \cdots \cdots \cdots \cdots \cdots \cdots \cdots \cdots \cdots \cdots \cdots \cdots \\
{\left[d_{n}, \tilde{\mu}\right] \leq-\ell^{n} \cdot v^{n i} \cdot \frac{1}{\mu^{i}} \forall i \in I,}
\end{array}\right.
$$

where $\tilde{\mu}=\left(\frac{1}{\mu^{1}}, \ldots, \frac{1}{\mu^{n}}\right)$.

From the last follows that

$$
\left\{\begin{array}{c}
{\left[d_{1}, \tilde{\mu}\right] \leq \min _{i \in I}\left(-\ell^{1} \cdot v^{1 i} \cdot \frac{1}{\mu^{i}}\right),} \\
\cdots \cdots \cdots \cdots \cdots \cdots \cdots \cdots \cdots \cdots \cdots \cdots \cdots \cdots \cdots \cdots \cdots \cdots \cdots \cdots \cdots \\
{\left[d_{n}, \tilde{\mu}\right] \leq \min _{i \in I}\left(-\ell^{n} \cdot v^{n i} \cdot \frac{1}{\mu^{i}}\right) .}
\end{array}\right.
$$

Let

$$
\tilde{d}_{i}=-\frac{1}{\ell^{i}} \cdot d_{i}(i \in I) .
$$

For this case we obtain that

$$
\left\{\begin{array}{c}
{\left[\tilde{d}_{1}, \tilde{\mu}\right] \geq \max _{i \in I} v^{1 i} \cdot \frac{1}{\mu^{i}}} \\
\cdots \cdots \cdots \cdots \cdots \cdots \cdots \cdots \cdots \cdots \cdots \cdots \cdots \cdots \cdots \\
{\left[\tilde{d}_{n}, \tilde{\mu}\right] \geq \max _{i \in I} v^{n i} \cdot \frac{1}{\mu^{i}} .}
\end{array}\right.
$$


Thus by this way the system of $n^{2}$ inequalities (24) is reduced to the system of $n$ superlinear inequalities.

\section{Results}

In the paper the following results are obtained:

-The necessary and sufficient conditions are derived for the optimality of the branch trajectories;

-The maximal growth rate is defined for the branches in the without loss case;

-The necessary and sufficient condition is derived for the existence of the equilibrium prices without loss;

-The form of the superdifferential is given for the utility function of the consumer;

-The conditions are defined for the reducing the system of $n^{2}$ linear inequalities to the system of $n$ superlinear inequalities of the same variables.

\section{References}

[1] Makarov V. L., Rubinov A. M., Mathematical Theory of the Economical Dynamics and Equilibrium, Moscow, Nauka, 1973, pp. 336.
[2] Rubinov A. M. Superlinear Multivalid Mappings and Their Applications to Economical-Mathematical Problems, L. Nauka, 1980.

[3] Rubinov A. M. Mathematical Models of the Expanded Reproduction Models. L. Nauka, 1973.

[4] Hamidov S. I. On a consumer problem, Journal of Pure and Applied Mathematics, Vol. 5, No. 6, 2016, pp. 205-210.

[5] Demyanov V. F., Rubinov A. M., Approximate Methods of Solution of the Extremal Problems, L., LSU, 1968, pp. 180.

[6] Demyanov V. F., Vasilyev L. V., Nondifferentiable Optimization, Moscow, Nauka, 1981, pp. 384.

[7] Fan Ji, On the Systems of Linear Inequalities, In the book "Linear Inequalities and Close Problems", Moscow, IL, 1969.

[8] Gantmakher F. R., The Theory of Matrices, Moscow, Nauka, 1988 , pp. 552.

[9] Rubinov A. M. Equilibrium Mechanisms for Effective and Development of Dynamic Models of Production and Exchange. Technical Cybernetics, 1968.

[10] Kleyner S. B. Production Functions, M., 1986. 